7th International Workshop on Astronomy and

Relativistic Astrophysics (IWARA 2016)

International Journal of Modern Physics: Conference Series

Vol. 45 (2017) 1760064 (8 pages)

(C) The Author(s)

DOI: $10.1142 / \mathrm{S} 2010194517600643$

\title{
Regge Phenomenology at LHC Energies
}

\author{
Carlos A. S. Bahia, Mateus Broilo and Emerson G. S. Luna \\ Instituto de Física, Universidade Federal do Rio Grande do Sul \\ 91501-970 Porto Alegre, Rio Grande do Sul, Brazil \\ andre.bahia@ufrgs.br,mateus.broilo@ufrgs.br, luna@if.ufrgs.br
}

Published 15 August 2017

\begin{abstract}
At high energies the Pomeron plays a crucial part in describing the soft interactions. In the light of LHC (Large Hadron Collider) data we perform a detailed analysis of protonproton $(p p)$ and antiproton-proton $(\bar{p} p)$ forward scattering data in order to determine the intercept and the slope of the soft Pomeron trajectory. This analysis is performed based on Regge theory using Born-level amplitudes. We investigate the role of the protonPomeron vertex form and of the nearest $t$-channel singularity. We give predictions for the total cross section and the ratio of the real part to the imaginary part of the elastic amplitude in $p p$ collisions at LHC and cosmic-ray energies.
\end{abstract}

Keywords: Pomeron, Regge Theory, Born-Level Amplitudes.

PACS numbers: 13.85.Dz, 13.85.Lg, 13.85.-t

\section{Introduction}

Recently the TOTEM experiment at the CERN LHC has released data on diffractive processes, which account for a substantial fraction of $p p$ and $\bar{p} p$ total cross sections at high energies ${ }^{1-6}$. Diffractive processes are characterized by the presence of one or more large rapidity gaps, which are associated with the exchange of the Pomeron. In the small $t$ domain these processes are described by Regge theory ${ }^{7}$, in which the behavior of the scattering amplitude is described by singularities in the complex plane of angular momentum $j$. In the simplest scenario diffractive processes are described by an isolated pole at $j=\alpha(t)$, resulting in an elastic amplitude

$$
A(s, t) \propto s^{\alpha(t)} .
$$

If more than one pole contributes we have different exchanges in the $t$-channel, and the elastic scattering amplitude is expressed as

This is an Open Access article published by World Scientific Publishing Company. It is distributed under the terms of the Creative Commons Attribution 4.0 (CC-BY) License. Further distribution of this work is permitted, provided the original work is properly cited. 


$$
A(s, t)=\sum_{i} \gamma_{i}(t) \eta_{i}(t) s^{\alpha_{i}(t)}
$$

where $\gamma_{i}(t)$ is the residue function and $\eta_{i}(t)$ is the signature factor. The magnitude of the Pomeron intercept $\alpha_{P}(0)$ plays a central role in Regge theory, since it is the pole with the largest intercept, originally $\alpha_{P}(0)=1$. However, in order to describe the observed increase of $p p$ and $\bar{p} p$ total cross sections with $s$, the Pomeron should have an intercept such that $\alpha_{P}(0)=1+\epsilon$ with $\epsilon>0$. This intercept value is possible also taking into account multi-Pomeron cuts in the $j$-plane. In this paper we determine the intercept and the slope of the Pomeron trajectory in the light of the recent LHC data, since these measurements provide a unique constraint on the Pomeron parameters. Moreover, the contribution of the Pomeron component to the $\chi^{2}$ is absolutely dominant in this energy regime.

\section{Born-Level Analysis}

In the large $s$-limit in a two-body scattering process $1+2 \rightarrow 3+4$ we consider, for single Regge exchange, an imaginary Born-level amplitude given by

$$
A_{i}(s, t)=i \beta_{i}^{2}(t)\left(\frac{s}{s_{0}}\right)^{\alpha_{i}(t)}
$$

where the index $i=P, f, \omega$ stands for the reggeon considered, $\beta_{i}(t)$ is the $t$-behavior of the reggeon-proton vertex (the residue function $\gamma_{i}$ factorizes as $\gamma_{i}(t)=\beta_{i}^{2}(t)$ ), and $s_{0}$ is a scale usually chosen to be of the order of $1 \mathrm{GeV}^{2}$. Degeneracies between the contributions of reggeons $f$ and $a$ and between $\omega$ and $\rho$ are implied since we are considering only three Regge exchange. Thus the complete imaginary Born-level amplitude is written by

$$
\operatorname{Im} A(s, t)=\operatorname{Im} A_{P}(s, t)+\operatorname{Im} A_{f}(s, t)+\tau \operatorname{Im} A_{\omega}(s, t),
$$

where $\tau=+1$ stands for proton-proton interaction and $\tau=-1$ for antiprotonproton. The total cross section is given by

$$
\sigma_{t o t}(s)=\frac{4 \pi}{s} \operatorname{Im} A(s, t=0)=X s^{\epsilon}+Y_{+} s^{-\eta_{+}}+\tau Y_{-} s^{-\eta_{-}},
$$

where

$$
X \equiv 4 \pi \beta_{P}^{2}(0) s_{0}^{-(1+\epsilon)} ; Y_{ \pm} \equiv \pm 4 \pi \beta_{ \pm}^{2}(0) s_{0}^{-\left(1-\eta_{ \pm}\right)} ; \alpha_{P}(0)=1+\epsilon
$$

and

$$
\alpha_{ \pm}(0)=1-\eta_{ \pm}
$$

The $\rho$ parameter is given by

$$
\rho(s)=\frac{\operatorname{Re} A(s, t=0)}{\operatorname{Im} A(s, t=0)}
$$


the connection between the real and imaginary parts of

$$
A(s, t)=\operatorname{Re} A(s, t)+i \operatorname{Im} A(s, t),
$$

at $t=0$, is obtained by means of dispersion relations: defining

$$
2 A_{ \pm}=A_{p p} \pm A_{\bar{p} p}
$$

these relations read ${ }^{8}$

$$
\begin{gathered}
\operatorname{Re} A_{+}(s, t=0)=K+s \tan \left[\frac{\pi}{2} \frac{d}{d \ln s}\right] \frac{\operatorname{Im} A_{+}(s, t=0)}{s}, \\
\operatorname{Re} A_{-}(s, t=0)=\tan \left[\frac{\pi}{2} \frac{d}{d \ln s}\right] \operatorname{Im} A_{-}(s, t=0),
\end{gathered}
$$

where $K$ is a subtraction constant (here defined to be zero). Within this formalism we obtain

$$
\rho(s) \sigma_{t o t}(s)=X s^{\epsilon} \tan \left(\frac{\pi}{2} \epsilon\right)+Y_{+} s^{-\eta_{+}} \tan \left(\frac{\pi}{2} \eta_{+}\right)+\tau Y_{-} s^{-\eta_{-}} \cot \left(\frac{\pi}{2} \eta_{-}\right) .
$$

The elastic differential cross section is expressed in terms of the complex amplitude $A(s, t)$,

$$
\frac{d \sigma}{d t}(s, t)=\frac{\pi}{s^{2}}|A(s, t)|^{2}
$$

we assume that the dispersion relations (11) and (12) are valid for $t \leq 1 \mathrm{GeV}^{2}$. The secondary reggeons $\left(a_{2}\right.$ and $\left.f_{2}\right)$ are taken to have an exponential form for the proton-reggeon vertex,

$$
\beta_{+}(t)=\beta_{+}(0) e^{r_{+} t}
$$

and to lie on an exchange-degenerate linear trajectory of form

$$
\alpha_{+}(t)=1-\eta_{+}+\alpha_{+}^{\prime} t
$$

Similarly, the secondary reggeons $\omega$ and $\rho$ are described by the parameters $\beta_{-}(0)$, $r_{-}, \eta_{-}$and $\alpha_{-}^{\prime}$. Concerning the proton-Pomeron vertex, we present some different variants in order to define different models. For models $B 1$ and $B 2$ we choose an exponential form for $\beta_{P}(t)$, namely ${ }^{9}$

$$
\beta_{P}(t)=\beta_{P}(0) e^{r_{P}^{(1)} t}
$$

For models $B 3$ and $B 4$ we choose a power-like form for $\beta_{P}(t)^{10}$,

$$
\beta_{P}(t)=\frac{\beta_{P}(0)}{\left(1-t / r_{P}^{(2)}\right)\left(1-t / r_{P}^{(3)}\right)}
$$


We also investigate different forms for the Pomeron trajectory. In model $B 1$ we adopt the linear trajectory

$$
\alpha_{P}(t)=\alpha_{P}(0)+\alpha_{P}^{\prime} t
$$

with $\alpha_{P}(0)=1+\epsilon$, whereas in the models $B 2, B 3$ and $B 4$ we introduce the nearest $t$-channel singularity in the trajectory, namely a two-pion loop insertion in the Pomeron exchange, which result in a nonlinear form for $\alpha_{P}(t)^{10,11}$,

$$
\alpha_{P}(t)=\alpha_{P}(0)+\alpha_{P}^{\prime} t-\frac{\left(\beta_{\pi}^{2} m_{\pi}\right)^{2}}{32 \pi^{3}} h\left(\frac{4 m_{\pi}^{2}}{|t|}\right)
$$

where $h(x)$ is written as

$$
h(x)=\frac{4}{x} F_{\pi}^{2}(t)\left[2 x-(1+x)^{3 / 2} \log \left(\frac{\sqrt{1+x}+1}{\sqrt{1+x}-1}\right)+\log \left(\frac{m^{2}}{m_{\pi}^{2}}\right)\right],
$$

with $x=4 m_{\pi}^{2} /|t|$. The coefficient $\beta_{\pi}$ specifies the $\pi \pi$ total cross section, but here we adopted the additive quark model relation $\beta_{\pi}=2 \beta_{P}(0) / 3$, and $F_{\pi}(t)$ is the form factor of the pion-Pomeron vertex,

$$
F_{\pi}(t)=1 /\left(1-t / r_{P}^{(2)}\right) .
$$

We consider a "pion cloud coupling to the Pomeron", where in model B2 and B3 the spatial distribution coefficient, $r_{P}^{(2)}$, is fixed in the square of the $\rho$ meson mass, $m_{\rho}=0.7754 \mathrm{GeV}$, and finally in model $B 4$ it is the ninth free parameter.

\section{Results and Concluding Remarks}

First, we carried out a global fit to high-energy forward $p p$ and $\bar{p} p$ scattering above $\sqrt{s}=10 \mathrm{GeV}$, namely, the total cross section $\sigma_{t o t}^{p p, \bar{p} p}$ and the ratio of the real to imaginary part of the forward scattering amplitude $\rho^{p p, \bar{p} p}$. We use data sets compiled and analyzed by the Particle Data Group ${ }^{12}$ as well as the recent data at LHC from the TOTEM Collaboration, with the statistic and systematic errors added in quadrature.

The TOTEM dataset includes the first and second measurements of the total $p p$ cross section at $\sqrt{s}=7 \mathrm{TeV}, \sigma_{\text {tot }}^{p p}=98.3 \pm 2.8$ (see Ref. ${ }^{1}$ ) and $\sigma_{\text {tot }}^{p p}=98.58 \pm 2,23$ (see Ref. ${ }^{2}$ ) (both using the optical theorem together with the luminosity provided by the CMS (see Ref. ${ }^{13}$ )), the luminosity-independent measurement at $\sqrt{s}=7$ $\mathrm{TeV}, \sigma_{\text {tot }}^{p p}=98.0 \pm 2.5$ (see Ref. ${ }^{3}$ ), the $\rho$-independent measurement at $\sqrt{s}=7$ $\mathrm{TeV}, \sigma_{\text {tot }}^{p p}=99.1 \pm 4.3$ (see Ref. ${ }^{3}$ ), and the luminosity-independent measurement at $\sqrt{s}=8 \mathrm{TeV}, \sigma_{t o t}^{p p}=101.7 \pm 2.9$ (see Ref. ${ }^{4}$ ). We include in the dataset the estimate for the $\rho$-parameter made by the TOTEM Collaboration in their $\rho$-independent measurement at $\sqrt{s}=7 \mathrm{TeV}$, namely $\rho^{p p}=0.145 \pm 0.091$ (see Ref. ${ }^{3}$ ) and for the first time at LHC the $\rho$-parameter was extracted via the Coulomb-nuclear interference at $\sqrt{s}=8 \mathrm{TeV}$, namely $\rho^{p p}=0.12 \pm 0.003$ (see Ref. ${ }^{5}$ ). We have also considered in our global fit the high-energy elastc $p p$ differential cross section data at LHC using the recent data given by the TOTEM Collaboration ${ }^{2}$ at $\sqrt{s}=7 \mathrm{TeV}$. 
In our analysis we have investigated the effects of some different form factors for the Pomeron-proton vertex as well as the effects of a linear Pomeron trajectory and the nearest $t$-channel singularity, namely the two-pion loop in the Pomeron nonlinear trajectory. The results of the fits to $\sigma_{t o t}$ and $\rho$ for both $p p$ and $\bar{p} p$ channels are displayed in Figure 1, together with the experimental data as well as the recent LHC data from the TOTEM Collaboration ${ }^{1-4}$ at $\sqrt{s}=7 \mathrm{TeV}$ and $8 \mathrm{TeV}$. We also show extrapolations of the total cross section in the region of cosmic-ray energies considering the data from Auger Collaboration ${ }^{14}$ at $\sqrt{s}=57 \mathrm{TeV}$ and Telescope Array ${ }^{15}$ at $\sqrt{s}=95 \mathrm{TeV}$. Although we are not considering in our fits the recent measurement of the $\sigma_{\text {tot }}$ from elastic scattering in $p p$ collisions at $\sqrt{s}=8 \mathrm{TeV}$ from the ATLAS Collaboration ${ }^{16}$ we decided at least to show the respectively datum by means of the discrepancy in comparison with the TOTEM results. For completeness the results of the fits to $d \sigma_{e l} / d|t|$ obtained using the Born-level models are displayed together with predictions at $\sqrt{s}=8 \mathrm{TeV}$ and $13 \mathrm{TeV}$ together with the recent experimental data ${ }^{2}$ at $\sqrt{s}=7 \mathrm{TeV}$ and the preliminary data ${ }^{6}$, still under analysis but not considered in our fitting procedure, at $\sqrt{s}=8 \mathrm{TeV}$. The values of the fitted parameters for all the five Born-level models are given in Table 1, where we also show the $\chi^{2} / D o F$ (degree of freedom).

Table 1. Values of the model parameters from the global fit to the scattering $p p$ and $\bar{p} p$ data.

\begin{tabular}{ccccc}
\hline & model B1 & model B2 & model B3 & model B4 \\
\hline$\beta_{P}(0)\left[\mathrm{GeV}^{-1}\right]$ & 1.9279 & 1.9271 & 1.9109 & 1.9249 \\
$r_{P}^{(1)}\left[\mathrm{GeV}^{-2}\right]$ & 4.3556 & 4.5084 & - & - \\
$r_{P}^{(2)}\left[\mathrm{GeV}^{2}\right]$ & - & - & 18.385 & 11.865 \\
$r_{P}^{(3)}\left[\mathrm{GeV}^{2}\right]$ & - & $m_{\rho}^{2}$ & $m_{\rho}^{2}$ & 12.6310 \\
$\epsilon$ & $0.9536 \times 10^{-1}$ & $0.9543 \times 10^{-1}$ & $0.9689 \times 10^{-1}$ & $0.9563 \times 10^{-1}$ \\
$\alpha_{P}^{\prime}\left[\mathrm{GeV}^{-2}\right]$ & $0.6906 \times 10^{-1}$ & $0.5132 \times 10^{-1}$ & 0.38859 & 0.53860 \\
$\beta_{+}(0)\left[\mathrm{GeV}^{-1}\right]$ & 3.4018 & 3.4010 & 3.3863 & 3.3992 \\
$\eta_{+}$ & 0.3243 & 0.3239 & 0.3173 & 0.3230 \\
$\beta_{-}(0)\left[\mathrm{GeV}^{-1}\right]$ & 2.4730 & 2.4719 & 2.4676 & 2.4721 \\
$\eta_{-}$ & 0.5258 & 0.5256 & 0.5249 & 0.5257 \\
\hline$\chi^{2} / D o F$ & 0.7954 & 0.7956 & 0.8298 & 0.8013 \\
\hline
\end{tabular}

In all the fits performed in this work we use a $\chi^{2}$ fitting procedure, assuming an interval $\chi^{2}-\chi_{\min }^{2}$ corresponding, in the case of normal errors, to the projection of the $\chi^{2}$ hyper-surface containing $90 \%$ of probability. In our models with 8 fitting parameters (and 9 fitting parameters) this corresponds to the interval $\chi^{2}-\chi_{\min }^{2}=$ $13.362(14.684)$. The $\chi^{2} / D o F$ for the best global fits lies in the range $[0.79,0.83]$ for 232 degrees of freedom. This good statistical result shows that our Born-level models are well suited for detailed predictions of the forward quantities to be measured at higher energies. In fact our predictions for $p p$ total cross section are statistically 
compatible with the AUGER result at $\sqrt{s}=57 \mathrm{TeV}$, namely

$$
\sigma_{\text {tot }}^{p p}=\left[133 \pm 13(\text { stat })_{-20}^{+17}(\text { syst }) \pm 16(\text { Glauber })\right] m b,
$$

(see Ref ${ }^{14}$ ), as well as with the Telescope Array result at $\sqrt{s}=95 \mathrm{TeV}$, namely

$$
\sigma_{\text {tot }}^{p p}=\left[170_{-44}^{+48}(\text { stat })_{-19}^{+17}(\text { syst })\right] m b,
$$

(see Ref. ${ }^{15}$ ). However it is worth noting that both results are model dependent, since they come from the conversion of the proton-air production cross section via a Glauber calculation. Moreover, as stressed by AUGER group, the total uncertainty of converting the proton-air to $p p$ cross section may be larger than the published. Clearly new results from AUGER and Telescope Array at higher energies would be extremely informative.
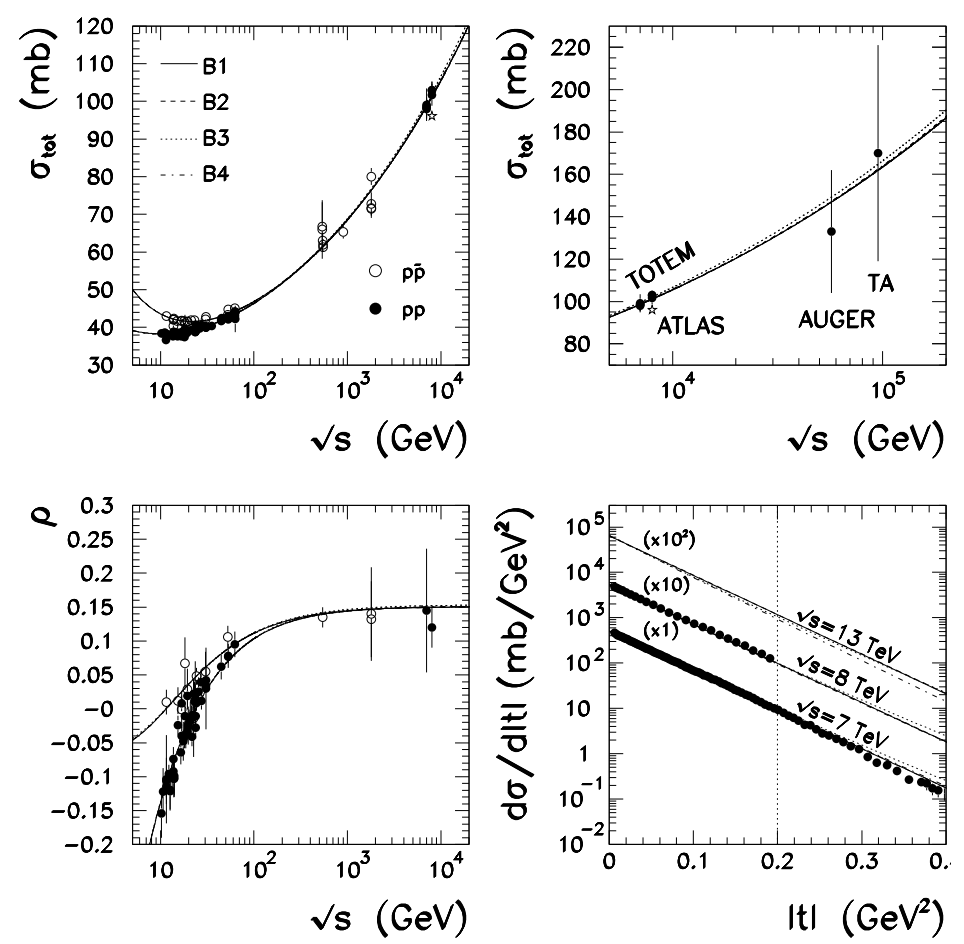

Fig. 1. Fits to the elastic scattering for both $p p$ and $p \bar{p}$ channels, and predictions to higher LHC energies and Cosmic-ray energies.

It is well known that Equation 3 leads to total cross sections which violates the Froissart-Martin bound ${ }^{17}$. The usual justification for using such Born-level models is that it is viewed as an effective scattering amplitude where the unitarity violation only occurs at extremely high energies far beyond LHC. One simple approach we 
could use in order to deal with unitarity violation is through eikonalized Regge amplitudes ${ }^{9}$, which is unitarized by construction:

$$
A_{e i k}(s, t)=i s \int_{0}^{\infty} d b b J_{0}(b \sqrt{-t})\left[1-e^{i \chi(s, b)}\right],
$$

where $J_{0}(x)$ is the Bessel function of zeroth order and the eikonal function defined as

$$
\chi(s, b)=\frac{1}{s} \int_{0}^{\infty} d q q J_{0}(b \sqrt{-t}) A_{B}(s, t) .
$$

Or still, as stressed by Cudell et al. ${ }^{18}$, we could place some physical constraints beyond single-pomeron exchange where it is expected that corrections at high energies through multiple-pomeron exchanges must tame the rise of total cross section according to the Froissart-Martin bound. Therefore the pomeron intercept could be interpreted as an effective one, which decreases as $\sqrt{s}$ increases. There are few information about multiple-pomeron contribution, but in general aspects the intercept for a two-pomeron exchange would be like $\alpha_{2 P}=2 \alpha_{P}-1$. We are carrying out both approaches in our model presented here and the results will soon be reported elsewhere.

\section{References}

1. G. Antchev et al., EPL 96, 21002 (2011).

2. G. Antchev et al., EPL 101, 21002 (2013).

3. G. Antchev et al., EPL 101, 21004 (2013).

4. G. Antchev et al., Phys. Rev. Lett. 111, 012001 (2013).

5. G. Antchev et al., arXiv:1610.00603, 527 (2016).

6. G. Antchev et al., Nuclear Physics B 899, 527 (2015).

7. V. Barone and E. Predazzi, High-Energy Particle Diffraction (Springer-Verlag, Berlin, 2002); S. Donnachie, G. Dosch, P. Landshoff, and O. Nachtmann, Pomeron Physics and $Q C D$ (Cambridge University Press, Cambridge, 2002); P. D. B. Collins, An introduction to Regge Theory and High-Energy Physics (Cambridge Monographs on Mathematical Physics, Cambridge Univ. Press, Cambridge, UK, 2009).

8. R. F. Ávila, E. G. S. Luna, and M. J. Menon, Phys. Rev. D B67, 054020 (2003). E. G. S. Luna and M. J. Menon, Phys. Lett. B 565, 123 (2003).

9. R. J. M. Covolan, J. Montanha, and K. Goulianos, Phys.Lett. B 389, 176 (1996).

10. V. A. Khoze, A. D. Martin, and M. G. Ryskin, Eur.Phys.J. C 18, 167 (2000). E. G. S. Luna, V. A. Khoze, A. D. Martin, and M. G. Ryskin, Eur.Phys.J. C 59, 1 (2009).

11. A. A. Anselm and V. N. Gribov, Phys.Lett. B 40, 487 (1972).

12. K. A. Olive et al., Chin. Phys. C 38, 090001 (2014).

13. CMS Collaboration, Performance Analysis Note CMS-PAS-EWK-10-004 (2010); CMS Collaboration, Detector Performance Note CMS-DP-2011-000 C (2011).

14. P.Abreu et al., Phys. Rev. Lett. 109, 062002 (2012).

15. R. U. Abbasi et al., Phys. Rev. D 92, 032007 (2015).

16. The ATLAS Collaboration, Phys. Lett. B 761, 158 (2016). 
17. M. Froissart, Phys. Rev. 123, 1053 (1961). A. Martin, Phys. Rev. 129, 1432 (1963).

18. J. R. Cudell, K. Kang, and S. K. Kim, Phys. Lett. B 395, 311 (1997). arXiv:hep-ph/9712235; Talk given at Conference: C97-06-10 p.114 (1997); arXiv:hepph/9701312; Presented at Conference: C96-06-03.3 p.134 (1997). J. R. Cudell, V. V. Ezhela, K. Kang, S. B. Lugovsky, and N. P. Tkachenko, arXiv:hep-ph/9812429; Presented at Conference: C98-09-17.1, p.62 (1998). 